\title{
Gender minority stress and access to health care services among transgender women and transfeminine people: results from a cross-sectional study in China
}

Yongjie Sha ${ }^{1 \dagger}$, Willa Dong ${ }^{2 \dagger}$, Weiming Tang ${ }^{1,3^{*}}$, Lingling Zheng ${ }^{4}, \mathrm{Xi} \mathrm{Huang}^{5}$, Kathryn E. Muessig $^{2}$ and Joseph D. Tucker ${ }^{1,3,6^{*}}$

\begin{abstract}
Background: Transgender and gender diverse individuals often face structural barriers to health care because of their gender minority status. The aim of this study was to examine the association between gender minority stress and access to specific health care services among transgender women and transfeminine people in China.
\end{abstract}

Methods: This multicenter cross-sectional study recruited participants between January 1st and June 30th 2020. Eligible participants were 18 years or older, assigned male at birth, not currently identifying as male, and living in China. Gender minority stress was measured using 45 items adapted from validated subscales. We examined access to health care services and interventions relevant to transgender and gender diverse people, including gender affirming interventions (hormones, surgeries), human immunodeficiency virus (HIV) and sexually transmitted infections (STIs) testing, pre-exposure prophylaxis (PrEP) and post-exposure prophylaxis (PEP). Multivariable regression was used to measure correlations between gender minority stress and access to health care service.

Results: Three hundred and twenty-four people completed a survey and data from 277 (85.5\%) people were analyzed. The mean age was 29 years old (standard deviation $[S D]=8)$. Participants used hormones $(118 / 277,42.6 \%)$, gender affirming surgery (26/277, 9.4\%), HIV testing (220/277, 79.4\%), STI testing (132/277, 47.7\%), PrEP (24/276, 8.7\%), and PEP (29/267, 10.9\%). Using gender affirming hormones was associated with higher levels of discrimination (adjusted odds ratio [aOR] 1.41,95\% confidence interval [Cl] 1.17-1.70) and internalized transphobia (aOR 1.06, 95\% Cl 1.00-1.12). STI testing was associated with lower levels of internalized transphobia (aOR 0.91, 95\%Cl 0.84-0.98).

Conclusions: Our data suggest that gender minority stress is closely related to using health services. Stigma reduction interventions and gender-affirming medical support are needed to improve transgender health.

Keywords: Transgender, Gender diverse, Gender minority stress, Sexual health, Gender-affirming care, China

*Correspondence: weiming_tang@med.unc.edu; jdtucker@med.unc.edu

${ }^{\dagger}$ Yongjie Sha and Willa Dong are co-first authors

1 University of North Carolina Project - China, 7 Lujing Road,

Guangzhou 510091, Guangdong, China

Full list of author information is available at the end of the article

\section{Background}

Many transgender and gender diverse individuals have unmet health needs [1]. Transgender individuals have a high prevalence of HIV infection [2]. Despite the high HIV prevalence, transgender and gender diverse individuals have low uptake of HIV prevention services [3, 4]. In addition, unmet needs for gender affirmation may 
increase sexual health risks for some transgender populations [5]. Gender affirmation refers to the process of receiving recognition and support for one's gender identity and expression, which is a key determinant of health and well-being for transgender and gender diverse people [1, 6-9]. Gender affirmation can involve medical interventions and/or changing one's name on legal documents $[10,11]$. Many transgender and gender diverse people lack access to medical gender affirmation or essential sexual health services. This disparity is related to stigma against these populations.

Stigma against transgender and gender diverse people refers to institutional, interpersonal, and individual processes that systemically disadvantage them socially, economically, and politically [5, 12, 13]. Transgender and gender diverse people may experience excess stress because of stigma based on identifying as a gender minority [14]. The gender minority stress and resilience (GMSR) model includes stressors related to discrimination, rejection, victimization, negative expectations for the future, internalized transphobia, and gender identity concealment [15]. Minority stress places gender and sexual minorities at risk for a number of negative physical and mental health outcomes [16, 17]. Research has suggested three major pathways that stigma and discrimination impact health: psychosocial stress, access to health and social resources, and violence and bodily harm [18]. Yet, these pathways are not always independent from each other in contributing to health outcomes [19]. For example, research among sexual minority men in the US has suggested that psychological stress has an impact on their engagement in HIV prevention [19]. Understanding the role of discrimination and psychological stress in access to sexual health prevention services is important because prevention is an essential part of comprehensive HIV control. For transgender populations in particular, discrimination and psychological stress can impact decisions to access gender affirmation medical care when other gender affirmation sources are restricted, which has powerful implications for their well-being [6]. However, no studies have assessed these associations in lowand middle-income countries (LMICs), including China.

Previous data suggested that Chinese transgender and gender diverse people experienced high levels of discrimination, victimization, and rejection $[20,21]$. Current laws and policies restrict transgender people's access to gender affirming services, including gender affirming medical care. Transgender and gender diverse people in China also lack protections against employment discrimination and sexual harassment [22]. Difficulties in finding gender affirming medical services push many to seek informal interventions or self-care for gender affirmation (hormones and surgeries) [22]. Additionally, Chinese transgender and gender diverse people may be less likely to test for HIV or sexually transmitted infections (STIs) compared to cisgender HIV key populations such as men who have sex with men (MSM) [23]. Few studies exist on pre-exposure prophylaxis (PrEP) or post-exposure prophylaxis (PEP) uptake in this population [24-26].

The purpose of this cross-sectional study was to examine gender minority stress and access to select health care services among transgender women and transfeminine people in China, focusing on access to gender affirming medical services and interventions (hereafter "gender affirming medical care") and sexual health services.

\section{Methods}

\section{Study design and population}

This cross-sectional study was conducted in nine cities across eight provinces in China. Study cities were selected because of their relatively large numbers of transgender women and transfeminine people and local communitybased organizations (CBOs) providing services to this population. Partnering CBOs shared study announcements on social media and in-person at outreach events and entertainment venues (mainly night clubs). Participants were recruited in-person before February 2020 and then in-person and online during and after February 2020 aligning with local regulations under the COVID-19 pandemic. Inclusion criteria were: 18 years old or older; assigned male at birth; currently identify as a woman, or as a non-binary or gender non-conforming person; and typically residing in one of the study cities.

\section{Data collection}

Participants completed the survey between January 1st and June 30th 2020. Eligible participants completed a self-administered computer-assisted survey. The survey collected data on sociodemographic information, gender identity, gender affirming medical care use (hormones, surgeries), HIV testing history, STI testing history (gonorrhea, chlamydia, syphilis, human papilloma virus [HPV] or condyloma acuminate, Herpes simplex virus 2 [HSV-2]), HIV serostatus, PrEP use, PEP use, gender minority stress and resilience, and sex work. We modified the two-step method to collect gender identity data [1]. Participants first selected their assigned sex at birth, and then gender identity from response options based on previous formative research and community expert feedback ("Transgender women", "CD", "Yao or TS", "Women", "Gender non-conforming, nonbinary", and "other, please describe") (unpublished). CD, TS, and Yao are local, reclaimed and contested identity terms [27]. Except for screening questions, participants were allowed to skip questions or choose "refuse to answer". Participants automatically received a unique eight-digit survey 
identification number after completing the online questionnaire (Sojump, Shanghai, China). All participants were required to contact $\mathrm{CBO}$ staff and verify questionnaire submission using their survey identification number to receive payment. Eligible participants who completed the survey received $\$ 15$.

Participants who visited CBOs that routinely offered HIV testing services were also asked whether they would like to receive HIV testing. CBO staff members obtained informed consent and performed the test following their standard protocol. Results were reported in an additional Sojump questionnaire, as well as the location and time of the test, survey identification number, and consent.

We restricted eligible responses as those that took at least $5 \mathrm{~min}$ as the survey hosted over 200 questions. Survey completion time was used to supplement other measures to prevent careless responses, such as attention checks [28]. CBOs also verified each questionnaire submission using survey identification numbers and contacted those suspected of submitting fraudulent responses.

\section{Measures}

\section{Sociodemographic}

Participants reported sociodemographic characteristics including age, ethnicity, housing, marital status, education, monthly income $(\mathrm{CNY} / \mathrm{USD}=6.5)$, sexual attraction, gender identity, gender identity disclosure, whether they currently live as the gender they identify as, and sex work involvement.

\section{Gender minority stress}

We adapted the subscales from the GMSR measure to assess gender minority stress [15]. These subscales measured participants' adverse experiences related to gender identity and perceptions about their own identity and transgender community. The measure includes seven factors: discrimination (5 items with total values ranging from 0 to 5, see Additional file 1: Online Appendix for a full list of items), rejection (6 items, $0-6$ ), victimization (6 items, 0-6), non-affirmation of gender identity (6 items, $0-18)$, internalized transphobia (8 items, $0-24)$, negative expectation for the future ( 9 items, $0-27$ ), and nondisclosure of gender identity (5 items, $0-15$ ). Items in the discrimination, rejection, and victimization subscales are scored 0 for "No" and 1 for "Yes". The non-affirmation of gender identity, internalized transphobia, negative expectation for the future, and nondisclosure of gender identity subscale items are scored on a 4-point Likert scale: 0 (strongly disagree), 1 (somewhat disagree), 2 (somewhat agree), and 3 (strongly agree). All subscales were modified to omit recency and whether or not the stressors were experienced before age 18 to reduce participant burden.

The subscales were adapted to the Chinese context following recommendations for cross-cultural adaptation of self-reported measures [29]. The subscales were translated into Chinese by two graduate students with expertise in public health and social science who were bilingual in English and Chinese, back translated into English by another bilingual public health graduate student, and reviewed by two academic and two community experts in transgender health in China. Ten cognitive interviews with transgender women and transfeminine people in two cities were conducted in Mandarin by a member of the study team (WD) and research assistants prior to pretesting. Feedback from the cognitive interviews were synthesized for each item. Scale items, instructions, and response options were modified based on these activities as well as prior formative and qualitative research (unpublished). Finally, pretesting with transgender women and transfeminine people was conducted prior to survey implementation.

\section{Access to health care services}

We included gender affirming medical care use (hormones, surgeries), HIV testing, STI testing, PrEP use, and PEP use to examine participants' access to health care services. In China, few hospitals offer gender affirming medical care, while HIV and STI testing can be accessed readily at hospitals, CBO-led testing clinics, or through self-testing kits purchased online. PrEP and PEP are only available at select infectious disease hospitals and require a prescription. Since PrEP was only approved in China in August 2020, participants in our survey likely accessed PrEP from informal sources or clinical trials. Similarly, transgender and gender diverse people in China may access gender affirming medical care informally due to low availability and high price. We did not distinguish between formal and informal access to these health care resources.

Lifetime HIV testing and PEP use were examined using single items with yes/no response options. STI testing in the past year was assessed where testing for gonorrhea, chlamydia, syphilis, HPV, or HSV-2 was counted as ever tested. For lifetime hormone intervention history, gender affirming surgery history, and PrEP use history, willingness was assessed in addition to yes/no response options. In this analysis, all responses other than ever use for gender affirming interventions and PrEP use were considered as never used.

\section{Statistical analysis}

Frequencies of categorical variables regarding sociodemographic characteristics and access to health care 
services were calculated as the proportion of participants. Mean scores of each gender minority subscales were calculated. Higher scores reflected a higher degree of minority stress. Cases with complete responses for the subscale of interest were included. Missing values were excluded from analysis. Multivariable regression (stepwise) was performed to assess associations between each gender minority stress subscale and each type of health care service. We adjusted for income, education, sexual attraction, and gender identity for analyses related to sexual health services access [30-33]. Additionally, we adjusted for whether the individual currently lives as their identified gender for models related to gender affirming medical care use. Adjusted odds ratios (aOR) were reported and the Wald 2-sided 95\% confidence interval (CI) was used. A 2-sided P value $<0.05$ was considered a statistically significant difference for all comparisons. All analyses were performed using R 3.6.3 (R Core Team).

This study was approved by institutional review boards at the University of North Carolina at Chapel Hill and Southern Medical University-Dermatology Hospital. All participants provided informed consent by checking a box on a self-administered online informed consent form indicating their agreement to participate in the study.

\section{Results}

Overall, 324 participants completed the survey. After excluding 28 submissions that were under five minutes, 18 unverified submissions from the same IP address, and one response from an individual under 18 years old, we included 277 participants for analysis.

\section{Sociodemographic characteristics of participants}

The mean age of the participants was 29 years old (Table 1). Most participants were never married (89\%), attracted to men (80.9\%), and self-identified as transgender women or women (58.8\%). For those who identified otherwise, 37 (13.4\%), 37 (13.4\%), and 39 (14.1\%) self-identified as "gender non-conforming, nonbinary", "CD", and "Yao or TS", respectively. The majority had disclosed their gender identity to at least one other person (207/275, 75.3\%), among whom most had disclosed to health workers $(155 / 207,74.9 \%)$. One hundred sixty-six participants $(60.1 \%)$ currently live as their self-identified gender for most of the time. Finally, one hundred sixteen (42.2\%) participants had ever exchanged sex for money in their lifetime.

\section{Access to health care services}

Table 2 presents data on participants' access to health care services. For gender affirming interventions, 42.6\% received hormone interventions while $9.4 \%$ underwent any gender affirming surgical procedures. Most
Table 1 Sociodemographic characteristics of transgender women and transfeminine people in China, December 2019June $2020(\mathrm{~N}=277)$

\begin{tabular}{|c|c|}
\hline Characteristics & $\mathrm{N}(\%)$ \\
\hline Age $(n=253)^{*}$ & $29.04(7.74)$ \\
\hline \multicolumn{2}{|l|}{ Housing } \\
\hline Live alone owned or rent & $163(58.8)$ \\
\hline Live with someone else & $62(22.4)$ \\
\hline Live at workplace or school & $38(13.7)$ \\
\hline Other & $14(5.1)$ \\
\hline \multicolumn{2}{|l|}{ Marital status $(n=276)^{*}$} \\
\hline Never married & $243(89.0)$ \\
\hline Engaged or married & $10(3.7)$ \\
\hline Separated or divorced & $23(8.4)$ \\
\hline Widowed & $0(0.0)$ \\
\hline \multicolumn{2}{|l|}{ Education } \\
\hline High school or below & $126(45.5)$ \\
\hline Some college & $68(24.5)$ \\
\hline College/Bachelors or above & $83(30.0)$ \\
\hline \multicolumn{2}{|l|}{ Annual income } \\
\hline No greater than USD\$5538 & $75(27.1)$ \\
\hline Greater than $\$ 5538$ and no greater than $\$ 11,077$ & $129(46.6)$ \\
\hline Greater than USD $\$ 11,077$ & $73(26.3)$ \\
\hline \multicolumn{2}{|l|}{ Sexual attraction } \\
\hline To women & $15(5.4)$ \\
\hline To men and women & $23(8.3)$ \\
\hline To men & $224(80.9)$ \\
\hline To no one & $3(1.1)$ \\
\hline Unsure or other & $12(4.3)$ \\
\hline \multicolumn{2}{|l|}{ Gender identity } \\
\hline Transgender woman & $104(37.5)$ \\
\hline$C D$ & $37(13.4)$ \\
\hline Yao orTS & $39(14.1)$ \\
\hline Women & $59(21.3)$ \\
\hline Gender non-conforming, nonbinary & $37(13.4)$ \\
\hline Other & $1(0.3)$ \\
\hline \multicolumn{2}{|c|}{ Currently live as self-identified gender for most of the time $(n=276)^{*}$} \\
\hline Yes & $166(60.1)$ \\
\hline No & $110(39.9)$ \\
\hline \multicolumn{2}{|l|}{ Disclosed identity $(n=275)^{*}$} \\
\hline Yes & $207(75.3)$ \\
\hline No & $68(24.7)$ \\
\hline \multicolumn{2}{|l|}{ Disclosed identity to health workers } \\
\hline Yes & $155(74.9)$ \\
\hline No & $52(25.1)$ \\
\hline \multicolumn{2}{|l|}{ Ethnicity } \\
\hline Han & $217(80.3)$ \\
\hline Other & $53(19.6)$ \\
\hline \multicolumn{2}{|l|}{ Lifetime sex work involvement } \\
\hline Yes & $116(42.2)$ \\
\hline No & 159 (57.8) \\
\hline
\end{tabular}


Table 1 (continued)

$\mathrm{CD}$, Yao, and TS are local transfeminine identity terms

*Numbers may not add up to 277 due to missing values

Table 2 Access to health care services among transgender women and transfeminine people in China, December 2019June $2020(N=277)$

\begin{tabular}{ll}
\hline Health care services & N (\%) \\
\hline Hormone intervention history & \\
Have or currently undergoing & $118(42.6)$ \\
Never & $159(57.4)$ \\
Gender affirming surgery & \\
Have or currently undergoing & $26(9.4)$ \\
Never & $251(90.6)$ \\
HIV testing & \\
No & $57(20.6)$ \\
Yes & \\
Lifetime HIV testing & $220(79.4)$ \\
Lifetime facility-based HIV testing & $190(86.4)$ \\
Lifetime HIV self-testing & $150(68.5)$ \\
STIs testing in the past year & \\
No & $145(52.3)$ \\
Yes & \\
Gonorrhea & $23(8.3)$ \\
Chlamydia & $15(5.4)$ \\
Syphilis & $124(44.8)$ \\
HPV & $33(11.9)$ \\
HSV2 & $8(2.9)$ \\
PrEP use & \\
Yes & $24(8.7)$ \\
No & $252(91.3)$ \\
YEP use & $29(10.9)$ \\
No & $238(89.1)$ \\
Living with HIV & $20(9.1)$ \\
\hline
\end{tabular}

participants (79.4\%) had ever received HIV testing while less than half $(47.7 \%)$ had ever tested for STIs. For PrEP and PEP, $8.7 \%$ and $10.9 \%$ had ever received these interventions, respectively. The HIV prevalence among those ever-tested was $9.1 \%(20 / 220)$.

\section{Experiences with gender minority stress}

Table 3 shows the mean scores of gender minority stress subscales. Additional file 1 reports scores for each item. Participants experienced high levels of non-affirmation of gender identity (mean, $M=11.92$ ) and scored the highest on item 2 ("I have difficulty being perceived as my gender.) $(M=2.10)$. Many participants $(80 \%)$ also reported being verbally harassed or teased (victimization subscale, item 1).

\section{Gender minority stress and access to health care services}

Table 4 presents the associations between gender minority stress and participants' access to health care services based on five different models with one model for each outcome. All variables included in the regression models are presented. Ever using hormones was associated with higher levels of discrimination (aOR 1.41, 95\%CI 1.171.70) and internalized transphobia (aOR 1.06, 95\% CI 1.00-1.12). Having undergone gender affirming surgery was associated with high levels of discrimination, with an adjusted odds ratio of 1.44 (95\% CI 1.06-1.97).

HIV testing was associated with lower levels of discrimination (aOR 0.70, 95\%CI 0.54-0.92). STI testing was associated with lower levels of internalized transphobia (aOR 0.91, 95\%CI 0.84-0.98) but higher levels of negative expectations for future (aOR 1.12, 95\%CI 1.04-1.19). PEP use was associated with higher levels of stress related to gender identity non-disclosure (aOR 1.19, 95\% CI 1.05-1.35).

Table 3 Gender minority stress subscale scores among transgender women and transfeminine people, January-June $2020(\mathrm{~N}=277)$

\begin{tabular}{llllr}
\hline Subscales (number of items) & Range & $\begin{array}{l}\text { Completion } \\
\text { N (\%) }\end{array}$ & Alpha* & $\begin{array}{l}\text { Total score } \\
\text { Mean } \pm \text { SD }^{\mathbb{*}}\end{array}$ \\
\hline Discrimination (5) & $0-5$ & $273(98.6)$ & 0.72 & $2.57 \pm 1.65$ \\
Rejection (6) & $0-6$ & $271(97.8)$ & 0.80 & $2.68 \pm 2.08$ \\
Victimization (6) & $0-6$ & $246(88.8)$ & 0.84 & $2.64 \pm 2.08$ \\
Non-affirmation of gender identity (6) & $0-18$ & $276(99.6)$ & 0.88 & $11.92 \pm 3.68$ \\
Internalized transphobia (8) & $0-24$ & $274(98.9)$ & 0.93 & $12.93 \pm 5.40$ \\
Negative expectations for future (9) & $0-27$ & $276(99.6)$ & 0.93 & $15.37 \pm 5.70$ \\
Non-disclosure of gender identity (5) & $0-15$ & $274(98.9)$ & 0.89 & $9.15 \pm 3.41$ \\
\hline
\end{tabular}

*Cronbach's alpha of internal consistency based on standardized items 
Table 4 Associations between gender identity stress and access to health care services among transgender women and transfeminine people in China, December 2019-June 2020

\begin{tabular}{|c|c|c|}
\hline Variables & aOR & $95 \% \mathrm{Cl}$ \\
\hline \multicolumn{3}{|c|}{ Hormone intervention history $(\mathrm{ref}=\text { never })^{\mathrm{a}}$} \\
\hline \multicolumn{3}{|c|}{ Have or currently undergoing } \\
\hline Discrimination & 1.41 & $1.17-1.70$ \\
\hline Internalized transphobia & 1.06 & $1.00-1.12$ \\
\hline \multicolumn{3}{|l|}{ Gender affirming surgery $(r e f=\text { never })^{a}$} \\
\hline \multicolumn{3}{|l|}{ Have or currently undergoing } \\
\hline Discrimination & 1.44 & $1.06-1.97$ \\
\hline Non-disclosure of gender identity & 0.87 & $0.77-0.99$ \\
\hline \multicolumn{3}{|l|}{ HIV testing $(r e f=n o)^{b}$} \\
\hline Discrimination & 0.70 & $0.54-0.92$ \\
\hline \multicolumn{3}{|l|}{ STIs testing in the past year $(r e f=n o)^{b}$} \\
\hline Internalized transphobia & 0.91 & $0.84-0.98$ \\
\hline Negative expectation for future & 1.12 & $1.04-1.19$ \\
\hline \multicolumn{3}{|l|}{ PEP use $(\text { ref }=\text { no })^{b}$} \\
\hline Non-disclosure of gender identity & 1.19 & $1.05-1.35$ \\
\hline \multicolumn{3}{|c|}{$\begin{array}{l}\text { a Models were adjusted with education, income, sexual attraction, gender } \\
\text { identity, and whether the individual currently live in self-identified gender for } \\
\text { most of the time }\end{array}$} \\
\hline${ }^{\mathrm{b}}$ Models were adjusted with education & & \\
\hline
\end{tabular}

\section{Discussion}

This study examined gender minority stress and access to health care resources among transgender women and transfeminine people in China. We identified low uptake of gender affirming medical care (hormones, surgeries), STI testing, PrEP and PEP. Discrimination and internalized transphobia are likely barriers to HIV and STI testing, though some gender minority stressors are also associated with higher uptake of some health care services (Fig. 1). More research on gender minority stress and discrimination among transgender and gender diverse people is needed. This study contributes to our understanding of the role of minority stress in engaging health care services.

We found low STI testing rates among study participants, which is consistent with prior studies among transgender women globally, including in China [23, 34]. Our findings suggest that internalized transphobia is a barrier to STI-but not HIV-testing among Chinese transgender women and transfeminine people. These results could be due to the fact that STI testing usually requires going to a hospital where providers may not be knowledgeable about transgender health while HIV testing is accessible at CBOs that often serve transgender and gender diverse people. There are ongoing efforts in China to expand STI testing among other key populations in China, but fewer programs focused on transgender and gender diverse people $[35,36]$.

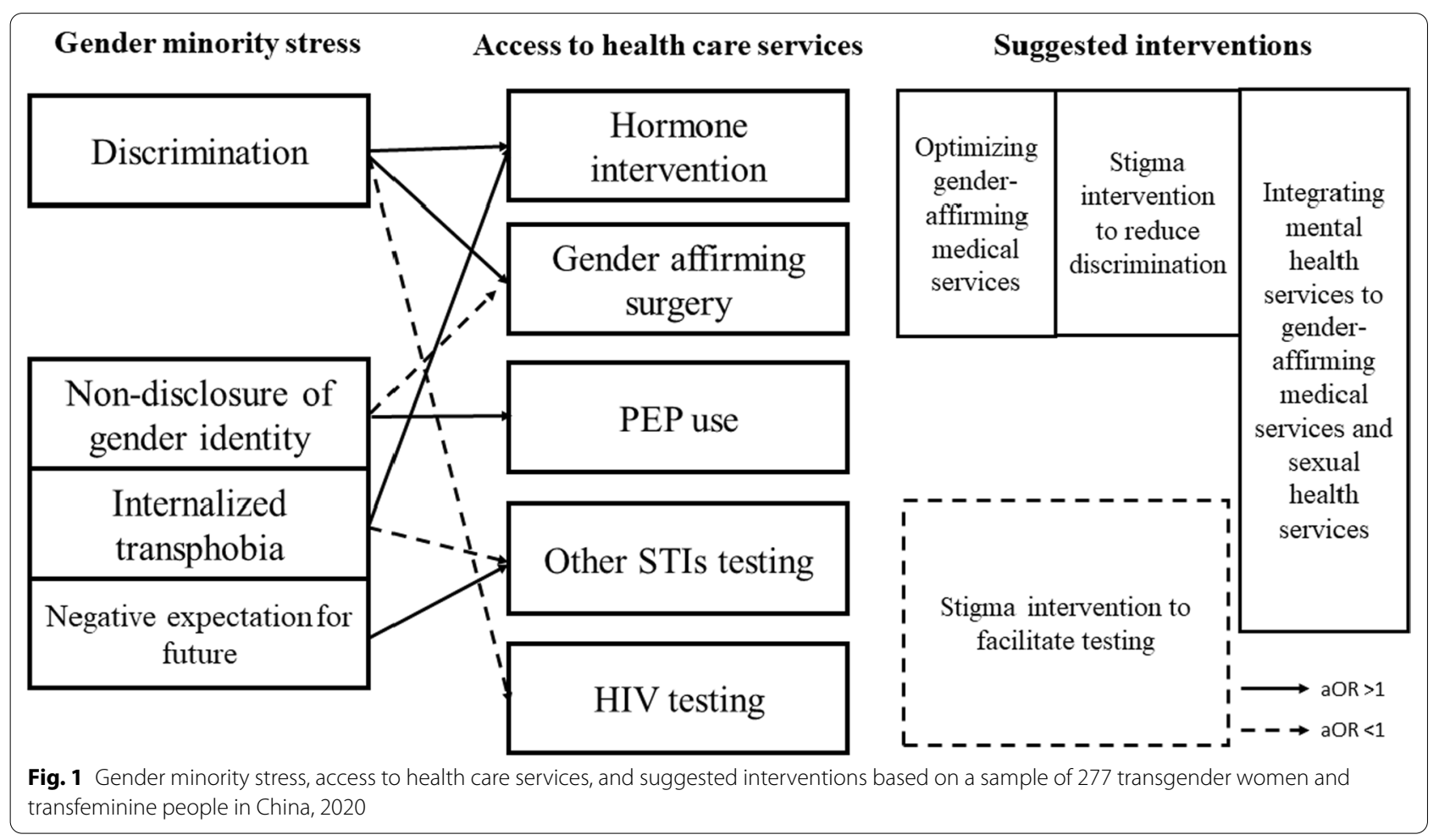


Interventions that address or take into consideration internalized transphobia such as self-testing or offering testing at venues reaching transgender and gender diverse people (CBOs, entertainment venues), training healthcare providers, and establishing transgenderfriendly clinics in the vein of MSM-friendly clinics may increase STI testing accessibility to Chinese transgender and gender diverse populations.

We found that transgender people who experienced discrimination and internalized transphobia were more likely to use gender affirming hormones. Previous data from the US suggested that these stressors can hinder accessing gender affirmation medical care [37]. This finding is aligned with the Gender Affirmation Framework suggesting discrimination and internalized transphobia may cause psychological distress that increase need for gender affirmation [6]. For example, those who experienced low visual conformity to dominant gender presentation norms are subject to discrimination and violence and might be more willing to receive hormone intervention [13]. However, this was not a longitudinal study and this relationship between discrimination and using gender affirming hormones could also be explained related to participants encountering discrimination in the process of getting care, informal access to hormones, or internalization of beauty standards. Future research is needed to assess the relationship between gender minority stress and gender affirmation services in resourcelimited settings. Research should also explore the ways Chinese transgender women and transfeminine people seek gender affirmation given the low availability of gender affirming medical care. More gender affirming medical care and other gender affirmation services (e.g., easier legal process for changing gender markers on documents) in China are needed. Multi-level interventions, including nationwide comprehensive gender identity education, gender-affirming health care capacity building, community empowerment and advocacy and psychological counseling, may also be helpful to reduce stress related to discrimination and internalized transphobia.

Similarly, the positive association between gender minority stressors and STI testing uptake may reflect that gender minority stress is closely associated with HIV prevention among study participants. Previous research on sexual minority men in the US suggested that psychological distress was associated with higher levels of HIV prevention engagement when felt stigma was low [19]. Although our study did not directly measure felt stigma, our findings support the hypothesis that minority stress could mediate the relationship between stigma and engagement in sexual health services [38].

\section{Research and policy implications}

Our findings have research and policy implications. First, our study suggests the importance of gender minority stressors to health outcomes given the associations we found with healthcare service access. More research is needed to examine the processes that connect stigma, gender minority stress, and health outcomes among transgender and gender diverse people in China. Second, stigma reduction measures targeting discrimination and internalized transphobia would be helpful to decrease barriers to HIV and STI testing. Few stigma reduction interventions are documented in the peer-reviewed literature due to the scarcity of research on transgender and gender diverse people and the political sensitivity of this group in China. Prior efforts from community-based organizations in China include transgender-affirmative mental health practice training for clinical professionals. Future interventions could include online support groups to reduce internalized transphobia and foster resilience; psychological counseling interventions to address internalized transphobia; training in cultural competence for primary care and sexual health physicians [13, 39, 40]. Third, interventions should address the high levels of minority stress related to accessing health services and interventions. Increasing the quality of gender affirming medical care, establishing standard operating protocols for medical professionals, and removing barriers to these services are important next steps. In terms of stressors associated with sexual health care access, we suggest integrating mental health services with sexual health interventions and care [41-43]. Sexual health services could integrate mental health components by directly providing counseling services, providing referrals to psychological services, and organizing community engagement activities for the transgender and gender diverse people [42, 44, 45].

\section{Limitations}

Our study should be read with limitations. First, this cross-sectional study is unable to establish temporality or explain associations. Additional research (longitudinal and qualitative) is needed on transgender and gender diverse people's experiences of health care service access in order to reduce minority stress. Second, most of the partnering community-based organizations were groups providing HIV testing services, as there were no transgender-specific CBOs that we knew of in most study cities. Collaborating organizations promoted the study through social media platforms and in-person outreach. This recruitment process could introduce selection bias. Compared to samples collected in previous studies [20,25], our participants have 
similar sociodemographic characteristics with regards to age, education, and income. However, due to the overall marginalization of transgender and gender diverse people in China, people who were older, less familiar with online survey, or less engaged in community-based HIV services may have been undersampled. A prior study in China [27] has demonstrated that it is feasible to use methods such as respondent-driven sampling to recruit participants. Building long-term, respectful partnerships with community-based organizations is key to reaching diverse participants across China. Third, our survey did not distinguish between informal and formal access to healthcare services such as hormone interventions. It is likely that differing gender minority stressors are relevant depending on access channels.

\section{Conclusions}

Gender minority stress is correlated with transgender gender diverse people's access to health care services in different ways. Discrimination and internalized transphobia are barriers to HIV and STI testing, while some gender minority stressors are associated with higher engagement in gender affirming medical care and sexual health prevention services. Future research in China should investigate the pathways that connect stigma, minority stress, and health outcomes to better understand transgender health inequalities and to inform interventions. Future policy is needed to prioritize providing gender-affirming medical services for Chinese transgender individuals and include mental health services in transgender health care delivery.

\begin{abstract}
Abbreviations
HIV: Human immunodeficiency virus; GMSR: Gender minority stress and resilience; US: The United States; LMIC: Low and middle-income country: STI: Sexually transmitted infection; MSM: Men who have sex with men; PrEP: Pre-exposure prophylaxis; PEP: Post-exposure prophylaxis; CBO: Communitybased organization; HPV: Human papilloma virus; HSV-2: Herpes simplex virus 2; CD: Cross-dresser; TS: Transsexual; aOR: Adjusted odds ratio; Cl: Confidence interval.
\end{abstract}

\section{Supplementary Information}

The online version contains supplementary material available at https://doi. org/10.1186/s12879-021-06782-5.

Additional file 1. Scores of gender minority stress subscales by item, $\mathrm{N}=277$, December 2019-June 2020.

\section{Acknowledgements}

The authors thank Guangxi Lvcheng Rainbow, Guiyang Caiai Benevolent Center, Jinan Rainbow, Nanjing Xingyou Working Group, Qingdao Qingtong Anti-AIDS Volunteer Service Center, Shenyang Sunshine Group, Trans Wellbeing Team, Wuhan LGBT Center, Yunnan Parallel, Zhitong Guangzhou LGBT Center for their help with recruitment. The authors also thank transgender community members in Beijing, Guangzhou and Shenyang for their input on survey adaptations.

\section{Authors' contributions}

YS conceptualized and designed the analysis, provided input on the analysis, interpreted findings, wrote the first draft and incorporated co-author feedback. WD conceptualized and designed the cross-sectional study, helped interpret findings, and participated in editing the article. WT oversaw data collection, conceptualized and designed the analysis, and provided input on the analysis. LZ led the data analysis. XH provided input to the development of cross-sectional survey, assisted with data collection, and participated in editing the article. KEM oversaw the development and implementation of the cross-sectional study. JDT oversaw data collection, helped interpret findings, and participated in editing the article. All authors read and approved the final manuscript.

\section{Funding}

This study was funded by the Graduate Tuition Incentive Scholarship and Dissertation Completion Fellowship, The Graduate School, The University of North Carolina at Chapel Hill; the STD/HIV T32 Predoctoral Training Program from the National Institute of Allergy and Infectious Diseases (Grant Number 5T32Al007001-42); the United States Department of Education FulbrightHays Doctoral Dissertation Research Abroad fellowship; the National Natural Science Foundation of China [Grant Numbers, 81903371]; the National Social Science Fund of China (No. 19CSH018); the National Key Research and Development Program of China [Grant Number 2017YFE0103800]; the National Institutes of Health [Grant Numbers R25 Al140495, R34MH109359, R34MH1 19963 and NIAID K24Al143471]; UNC Center for AIDS Research [Grant Number NIAID 5P30AI050410]. The funders had no role in study design, data collection, and analysis, decision to publish, or preparation of the manuscript.

\section{Availability of data and materials}

The data sets used and/or analyzed during the current study may be made available upon reasonable written request to the corresponding author.

\section{Declarations}

\section{Ethics approval and consent to participate}

This study was approved by institutional review boards at the University of North Carolina at Chapel Hill and Southern Medical University-Dermatology Hospital. All participants provided informed consent by checking a box on a self-administered online informed consent form indicating their agreement to participate in the study. All stages of the research were carried out in accordance with the current guidelines and regulations of ethics in research with human beings.

\section{Consent for publication \\ Not applicable.}

\section{Competing interests}

The authors declare that they have no competing interests. Dr. Weiming Tang is an associate editor of BMC Infectious Diseases, and Dr. Joseph D. Tucker is a section editor of BMC Infectious Diseases.

\section{Author details}

${ }^{1}$ University of North Carolina Project - China, 7 Lujing Road, Guangzhou 510091, Guangdong, China. ${ }^{2}$ Gillings School of Global Public Health, University of North Carolina at Chapel Hill, Chapel Hill, NC, USA. ${ }^{3}$ School of Medicine, University of North Carolina at Chapel Hill, Chapel Hill, NC, USA. ${ }^{4}$ Guangzhou Women and Children's Medical Center, Guangzhou, China. ${ }^{5}$ Trans Well-being Team, Guangzhou, China. ${ }^{6}$ Faculty of Infectious and Tropical Diseases, London School of Hygiene \& Tropical Medicine, London, UK.

Received: 3 June 2021 Accepted: 29 September 2021

Published online: 14 October 2021

\author{
References \\ 1. Reisner SL, Poteat T, Keatley J, et al. Global health burden and needs of \\ transgender populations: a review. Lancet. 2016;388(10042):412-36.
}


2. Van Gerwen OT, Jani A, Long DM, et al. Prevalence of sexually transmitted infections and human immunodeficiency virus in transgender persons: a systematic review. Transgend Health. 2020;5(2):90-103.

3. Vaitses Fontanari AM, Zanella Gl, Feijó M, et al. HIV-related care for transgender people: a systematic review of studies from around the world. Soc Sci Med. 2019;230:280-94.

4. Fisher $C B$, Fried $A L$, Desmond $M$, et al. Perceived barriers to HIV prevention services for transgender youth. LGBT Health. 2018;5(6):350-8.

5. AVAC. No data no more: manifesto to align HIV prevention research with trans and gender diverse realities. 2021. https://www.avac.org/no-data-nomore. Accessed 12 Sep 2021.

6. Sevelius JM. Gender affirmation: a framework for conceptualizing risk behavior among transgender women of color. Sex Roles. 2013;68(11-12):675-89.

7. Wernick JA, Busa S, Matouk K, et al. A systematic review of the psychological benefits of gender-affirming surgery. Urol Clin North Am. 2019;46(4):475-86

8. Baker KE, Wilson LM, Sharma R, et al. Hormone therapy, mental health, and quality of life among transgender people: a systematic review. J Endocr Soc 2021;5(4):bvab011.

9. Glynn TR, Gamarel KE, Kahler CW, et al. The role of gender affirmation in psychological well-being among transgender women. Psychol Sex Orientat Gend Divers. 2016;3(3):336-44.

10. Reback CJ, Clark K, Holloway IW, et al. Health disparities, risk behaviors and healthcare utilization among transgender women in Los Angeles county: a comparison from 1998-1999 to 2015-2016. AIDS Behav. 2018;22(8):2524-33.

11. Jiang $H$, Wei $X, Z$ Zhu $X$, et al. Transgender patients need better protection in China. Lancet. 2014;384(9960):2109-10.

12. King WM, Hughto JMW, Operario D. Transgender stigma: a critical scoping review of definitions, domains, and measures used in empirical research. Soc Sci Med. 2020:250:112867.

13. White Hughto JM, Reisner SL, Pachankis JE. Transgender stigma and health: a critical review of stigma determinants, mechanisms, and interventions. Soc Sci Med. 2015;147:222-31.

14. Meyer $I H$. Prejudice, social stress, and mental health in lesbian, gay, and bisexual populations: conceptual issues and research evidence. Psychol Bull. 2003;129(5):674-97.

15. Testa RJ, Habarth J, Peta J, et al. Development of the gender minority stress and resilience measure. Psychol Sex Orientat Gend Divers. 2015;2(1):65-77.

16. Reisner SL, White Hughto JM, Gamarel KE, Keuroghlian AS, Mizock L, Pachankis JE. Discriminatory experiences associated with posttraumatic stress disorder symptoms among transgender adults. J Couns Psychol. 2016;63(5):509-19.

17. Lick DJ, Durso LE, Johnson KL. Minority stress and physical health among sexual minorities. Perspect Psychol Sci. 2013;8(5):521-48.

18. Health Affairs Blog. Discrimination: a social determinant of health inequities. 2020. https://www.healthaffairs.org/do/10.1377/hblog20200220.518458/ full/?_cf_chl_captcha_tk_=pmd_dJ1tgbURNXGzdckl_o7F5bLtT9U VMpG91bSbnbepl6w-1631356056-0-gaNtZGzNA2WjcnBszQ1I. Accessed 12 Sept 2021

19. Krueger EA, Holloway IW, Lightfoot M, Lin A, Hammack PL, Meyer IH. Psychological distress, felt stigma, and HIV prevention in a national probability sample of sexual minority men. LGBT Health. 2020;7(4):190-7.

20. She R, Mo PK, MaT, et al. Impact of minority stress and poor mental health on sexual risk behaviors among transgender women sex workers in Shenyang, China. AIDS Behav. 2021;25(6):1790-9.

21. Zhang J, Lo HH, Au AM. The buffer of resilience in the relations of genderrelated discrimination, rejection, and victimization with depression among Chinese transgender and gender non-conforming individuals. J Affect Disord. 2021:283:335-43.

22. UNDP and China Women's University. Legal gender recognition in China: a legal and policy review. 2018. https://www.cn.undp.org/content/china/en/ home/library/democratic_governance/legal-gender-recognition-in-china-a-legal-and-policy-review-.html. Accessed 2 Aug 2021.

23. Best J, Tang W, Zhang Y, et al. Sexual behaviors and HIV/syphilis testing among transgender individuals in China: implications for expanding HIV testing services. Sex Transm Dis. 2015;42(5):281-5.

24. Xu J, Tang W, Zhang F, et al. PrEP in China: choices are ahead. Lancet HIV. 2020;7(3):e155-7.

25. Wang Z, Lau JTF, Yang $X$, et al. Acceptability of daily use of free oral preexposure prophylaxis (PrEP) among transgender women sex workers in Shenyang, China. AIDS Behav. 2017;21(12):3287-98.
26. Yan L, Yan Z, Wilson E, et al. Awareness and Willingness to use HIV preexposure prophylaxis (PrEP) among trans women in China: a communitybased survey. AIDS Behav. 2020;25(3):866-74.

27. Yan ZH, Lin J, Xiao WJ, et al. Identity, stigma, and HIV risk among transgender women: a qualitative study in Jiangsu Province, China. Infect Dis Poverty. 2019;8(1):94.

28. Meade AW, Craig SB. Identifying careless responses in survey data. Psychol Methods. 2012;17(3):437-55

29. Beaton DE, Bombardier C, Guillemin F, et al. Guidelines for the process of cross-cultural adaptation of self-report measures. Spine. 2000;25(24):3186-91.

30. Andersen RM, Davidson PL. Improving access to care in America: individual and contextual indicators. In: Anderson R, Rice T, Kominski J, editors. Changing the US health care system: Key issues in health services, policy and management. San Francisco: Jossey-Bass; 2001. p. 3-30.

31. Ross C, Wu C. The links between education and health. Am Sociol Rev. 1995;60(5):719-45.

32. Alencar Albuquerque G, de Lima GC, da Silva QG, et al. Access to health services by lesbian, gay, bisexual, and transgender persons: systematic literature review. BMC Int Health Hum Rights. 2016;16:2.

33. Hiestand KR, Horne SG, Levitt HM. Effects of gender identity on experiences of healthcare for sexual minority women. J LGBT Health Res. 2007:3(4):15-27.

34. Hakim AJ, Iwamoto C, Badman SG, et al. High prevalence of chlamydia and gonorrhea and the need for STI testing among men who have sex with men and transgender women in Papua New Guinea. Sex Transm Dis. 2021;48(2):109-17.

35. Wu D, Zhou Y, Yang N, et al. Social media-based secondary distribution of HIV/syphilis self-testing among Chinese men who have sex with men. Clin Infect Dis. 2020. https://doi.org/10.1093/cid/ciaa825.

36. Yang F, Zhang TP, Tang W, et al. Pay-it-forward gonorrhoea and chlamydia testing among men who have sex with men in China: a randomised controlled trial. Lancet HIV. 2020;20(8):976-82.

37. Goldenberg T, Kahle EM, Stephenson R. Stigma, resilience, and health care use among transgender and other gender diverse youth in the United States. Transgend Health. 2020;5(3):173-81.

38. Hatzenbuehler ML. How does sexual minority stigma "get under the skin"? A psychological mediation framework. Psychol Bull. 2009;135(5):707-30.

39. Lelutiu-Weinberger C, Pachankis JE. Acceptability and preliminary efficacy of a lesbian, gay, bisexual, and transgender-affirmative mental health practice training in a highly stigmatizing national context. LGBT Health. 2017:4(5):360-70.

40. Logie CH, Dias LV, Jenkinson J, et al. Exploring the potential of participatory theatre to reduce stigma and promote health equity for lesbian, gay, bisexual, and transgender (LGBT) people in Swaziland and Lesotho. Health Educ Behav. 2019:46(1):146-56

41. Safren SA, Thomas B, Biello KB, et al. Strengthening resilience to reduce HIV risk in Indian MSM: a multicity, randomised, clinical efficacy trial. Lancet Glob Health. 2021;9(4):e446-55.

42. Shaikh S, Mburu G, Arumugam V, et al. Empowering communities and strengthening systems to improve transgender health: outcomes from the Pehchan programme in India. J Int AIDS Soc. 2016;19(3 Suppl 2):20809.

43. Wylie K, Knudson G, Khan Sl, et al. Serving transgender people: clinical care considerations and service delivery models in transgender health. Lancet. 2016;388(10042):401-11.

44. Singh RJ, Sarna A, Schensul JJ, et al. A multilevel intervention to reduce stigma among alcohol consuming men living with HIV receiving antiretroviral therapy: findings from a randomized control trial in India. AIDS. 2020;34(Suppl 1):S83-92

45. Collins PY, Velloza J, Concepcion T, et al. Intervening for HIV prevention and mental health: a review of global literature. J Int AIDS Soc. 2021;24(Suppl 2):e25710.

\section{Publisher's Note}

Springer Nature remains neutral with regard to jurisdictional claims in published maps and institutional affiliations. 